\title{
Pullback Attractors for Nonautonomous 2D-Navier-Stokes Models with Variable Delays
}

\author{
Xiaoli Liu and Yejuan Wang \\ School of Mathematics and Statistics, Lanzhou University, Lanzhou 730000, China \\ Correspondence should be addressed to Yejuan Wang; wangyj@lzu.edu.cn
}

Received 5 January 2013; Accepted 30 March 2013

Academic Editor: de Dai

Copyright (c) 2013 X. Liu and Y. Wang. This is an open access article distributed under the Creative Commons Attribution License, which permits unrestricted use, distribution, and reproduction in any medium, provided the original work is properly cited.

Using a method based on the concept of the Kuratowski measure of the noncompactness of a bounded set as well as some new estimates of the equicontinuity of the solutions, we prove the existence of a unique pullback attractor in higher regularity space for the multivalued process associated with the nonautonomous 2D-Navier-Stokes model with delays and without the uniqueness of solutions.

\section{Introduction}

It is well known that the Navier-Stokes equations are very important in the understanding of fluids motion and turbulence. These equations have been studied extensively over the last decades (see [1-3], and the references cited therein). Recently, Caraballo and Real [4] considered global attractors for functional Navier-Stokes models with the uniqueness of solutions and for the delay, so that a wide range of hereditary characteristics (constant or variable delay, distributed delay, etc.) can be treated in a unified way. Very recently, MarínRubio and Real [5] used the theory of multivalued dynamical system to establish the existence of attractors for the 2DNavier-Stokes model with delays, when the forcing term containing the delay is sublinear and only continuous.

For the study of asymptotic behavior for functional partial differential equations without the uniqueness of solutions, as far as we know, not many papers have been published. However, some results in the finite dimensional context can be found in [6,7] (see also [8-10] for some preliminary and interesting results on the structure of the attractors for ordinary differential delay systems).

The pullback attractor is a possible approach to define an "attractor" for the nonautonomous dynamical systems, the long time behavior of nonautonomous dynamical systems is an interesting and challenging problem; see, for example, [1119], and so forth. The purpose of our current paper is to study existence of pullback attractors for the following functional Navier-Stokes problem:

$$
\begin{aligned}
& \frac{\partial u}{\partial t}-v \Delta u+\sum_{i=1}^{2} u_{i} \frac{\partial u}{\partial x_{i}} \\
&=f(t, u(t-\rho(t)))-\nabla p+g(t) \quad \text { in }(\tau,+\infty) \times \Omega, \\
& \operatorname{div} u=0 \quad \text { in }(\tau,+\infty) \times \Omega, \\
& u=0 \quad \text { on }(\tau,+\infty) \times \Gamma, \\
& u(\tau+t, x)=\phi(t, x), \quad t \in[-h, 0], x \in \Omega,
\end{aligned}
$$

where $\Omega \subset \mathbb{R}^{2}$ is an open bounded set with regular boundary $\Gamma, v>0$ is the kinematic viscosity, $u$ is the velocity field of the fluid, $p$ is the pressure, $\tau \in \mathbb{R}$ is the initial time, $g$ is a nondelayed external force field, $f$ is another external force term and contains some memory effects during a fixed interval of time of length $h>0, \rho$ is an adequate given delay function, and $\phi$ the initial datum on the interval $[-h, 0]$.

Using the technique of measure of noncompactness, noting that all norms on finite dimensional spaces are equivalent, we apply the new method to check the pullback $\omega$-limit compactness given in [20] and then get the existence of the pullback attractors in $C_{V}$. 
We consider the following usual abstract spaces:

$$
\mathscr{V}=\left\{u \in\left(C_{0}^{\infty}(\Omega)\right)^{2}: \operatorname{div} u=0\right\},
$$

where $H=$ the closure of $\mathscr{V}$ in $\left(L^{2}(\Omega)\right)^{2}$ with norm $|\cdot|$ and inner product $(\cdot, \cdot)$, where for $u, v \in\left(L^{2}(\Omega)\right)^{2}$,

$$
(u, v)=\sum_{j=1}^{2} \int_{\Omega} u_{j}(x) v_{j}(x) d x
$$

where $V=$ the closure of $\mathscr{V}$ in $\left(H_{0}^{1}(\Omega)\right)^{2}$ with norm $\|\cdot\|$ and associated scalar product $((\cdot, \cdot))$, where for $u, v \in\left(H_{0}^{1}(\Omega)\right)^{2}$,

$$
((u, v))=\sum_{i, j=1}^{2} \int_{\Omega} \frac{\partial u_{j}}{\partial x_{i}} \frac{\partial v_{j}}{\partial x_{i}} d x
$$

Note that $V \subset H \equiv H^{\prime} \subset V^{\prime}$, where the injections are dense and compact. We will use $\|\cdot\|_{*}$ for the norm in $V^{\prime}$ and $\langle\cdot, \cdot\rangle$ for the duality pairing between $V$ and $V^{\prime}$.

Define the trilinear form $b$ on $V \times V \times V$ by

$$
b(u, v, w)=\sum_{i, j=1}^{2} \int_{\Omega} u_{i} \frac{\partial v_{j}}{\partial x_{i}} w_{j} d x, \quad \forall u, v, w \in V .
$$

Now, let us establish some assumptions for (1).

We assume that the given delay function satisfies $\rho \in$ $C^{1}(\mathbb{R} ;[0, h])$, and there exists a constant $\rho_{*}$ satisfying

$$
\rho^{\prime}(t) \leqslant \rho_{*}<1, \quad \forall t \in \mathbb{R} .
$$

Furthermore, we suppose that $f$ and $g$ satisfy the following assumptions:

(H1) $f(\cdot, v): \mathbb{R} \rightarrow H$ is measurable for all $v \in H$,

(H2) $f(t, \cdot): H \rightarrow H$ is continuous for all $t \in \mathbb{R}$,

(H3) there exist positive constants $k_{1}, k_{2}$ such that for any $v \in H$,

$$
|f(t, v)|^{2} \leqslant k_{1}^{2}+k_{2}^{2}|v|^{2}, \quad \forall t \in \mathbb{R},
$$

(H4) there exists a fixed $\delta_{0}>0$ such that for any $\delta \in\left(0, \delta_{0}\right)$, the external force $g \in L_{\text {loc }}^{2}(\mathbb{R} ; H)$ satisfies

$$
\int_{-\infty}^{t}|g(r)|^{2} e^{\delta r} d r<\infty, \quad \forall t \in \mathbb{R} .
$$

Set $A: V \rightarrow V^{\prime}$ as $\langle A u, v\rangle=((u, v)), B: V \times V \rightarrow V^{\prime}$ by $\langle B(u, v), w\rangle=b(u, v, w)$, for all $u, v, w \in V$. Denote by $P$ the corresponding orthogonal projection $P:\left(L^{2}(\Omega)\right)^{2} \rightarrow H$. We further set $A:=-P \Delta$. The Stokes operator $A$ is self-adjoint and positive from $D(A)=V \cap\left(H^{2}(\Omega)\right)^{2}$ to $H$. The inverse operator is compact. Excluding the pressure, the system (1) can be written in the form

$$
\begin{aligned}
\frac{d}{d t} u(t) & +v A u(t)+B(u(t), u(t)) \\
= & f(t, u(t-\rho(t)))+g(t) \quad \text { in } \mathscr{D}^{\prime}\left(\tau,+\infty ; V^{\prime}\right), \\
& u(\tau+t)=\phi(t), \quad t \in[-h, 0], x \in \Omega .
\end{aligned}
$$

\section{Preliminaries}

Let $X$ be a complete metric space with metric $d_{X}(\cdot, \cdot)$, and denote by $\mathscr{P}(X)$ the class of nonempty subsets of $X$. As usual, let us denote by $H_{X}^{*}(\cdot, \cdot)$ the Hausdorff semidistance between $A$ and $B$, which are defined by

$$
H_{X}^{*}(A, B)=\sup _{a \in A} \operatorname{dist}_{X}(a, B)
$$

where $\operatorname{dist}_{X}(a, B)=\inf _{b \in B} d_{X}(a, b)$. Finally, denote by $\mathcal{N}(A$, $r)$ the open neighborhood $\left\{y \in X \mid \operatorname{dist}_{X}(y, A)<r\right\}$ of radius $r>0$ of a subset $A$ of a Banach space $X$.

Definition 1. A family of mappings $U(t, \tau): X \rightarrow \mathscr{P}(X)$, $t \geqslant \tau, \tau \in \mathbb{R}$ is called to be a multivalued process (MVP in short) if it satisfies

(1) $U(\tau, \tau) x=\{x\}$, for all $\tau \in \mathbb{R}, x \in X$;

(2) $U(t, s) U(s, \tau) x=U(t, \tau) x$, for all $t \geqslant s \geqslant \tau, \tau \in$ $\mathbb{R}, x \in X$.

Let $\mathscr{D}$ be a nonempty class of parameterized sets $\mathscr{D}=$ $\{D(t)\}_{t \in \mathbb{R}} \subset \mathscr{P}(X)$.

Definition 2. Let $\{U(t, \tau)\}$ be a multivalued process on $X$. One says that $\{U(t, \tau)\}$ is

(1) pullback $\mathscr{D}$-dissipative, if there exists a family $\mathbb{Q}=$ $\{Q(t)\}_{t \in \mathbb{R}} \in \mathscr{D}$, so that for any $\mathscr{B}=\{B(t)\}_{t \in \mathbb{R}} \in \mathscr{D}$ and each $t \in \mathbb{R}$, there exists a $t_{0}=t_{0}(\mathscr{B}, t) \in \mathbb{R}^{+}$such that

$$
U(t, t-s) B(t-s) \subset Q(t), \quad \forall s \geqslant t_{0} ;
$$

(2) pullback $\mathscr{D}$-limit-set compact with respect to each $t \epsilon$ $\mathbb{R}$, if for any $\mathscr{B}=\{B(t)\}_{t \in \mathbb{R}} \in \mathscr{D}$ and $\varepsilon>0$, there exists a $t_{1}=t_{1}(\mathscr{B}, t, \varepsilon) \in \mathbb{R}^{+}$such that

$$
k\left(\bigcup_{s \geqslant t_{1}} U(t, t-s) B(t-s)\right) \leqslant \varepsilon
$$

where $k$ is the Kuratowski measure of noncompactness.

Definition 3. A family of nonempty compact subsets $\mathscr{A}=$ $\{A(t)\}_{t \in \mathbb{R}} \subset \mathscr{P}(X)$ is called to be a pullback $\mathscr{D}$-attractor for the multivalued process $\{U(t, \tau)\}$, if it satisfies

(1) $\mathscr{A}=\{A(t)\}_{t \in \mathbb{R}}$ is invariant; that is,

$$
U(t, \tau) A(\tau)=A(t), \quad \forall t \geqslant \tau, \tau \in \mathbb{R},
$$

(2) $\mathscr{A}$ is pullback $\mathscr{D}$-attracting; that is, for every $\mathscr{B} \in \mathscr{D}$ and any fixed $t \in \mathbb{R}$,

$$
\lim _{s \rightarrow+\infty} H_{X}^{*}(U(t, t-s) B(t-s), A(t))=0 .
$$

Let $X, Y$ be two Banach spaces, and let $X^{*}, Y^{*}$ be their dual spaces, respectively. We also assume that $X$ is a dense subspace of $Y$, the injection $i: X \hookrightarrow Y$ is continuous, and its adjoint $i^{*}: Y^{*} \hookrightarrow X^{*}$ is densely injective. 
Theorem 4 (see $[21,22]$ ). Let $X, Y$ be two Banach spaces satisfy the previous assumptions, and let $\{U(t, \tau)\}$ be a multivalued process on $X$ and $Y$, respectively. Assume that $\{U(t, \tau)\}$ is upper semicontinuous or weak upper semicontinuous on $Y$. If for fixed $t \geqslant \tau, \tau \in \mathbb{R}, U(t, \tau)$ maps compact subsets of $X$ into bounded subsets of $\mathscr{P}(X)$, then $U(t, \tau)$ is norm-to-weak upper semicontinuous on $X$.

By slightly modifying the arguments of Theorem 3.4 and Remark 3.9 in [21], we have the following.

Theorem 5. Let $X$ be a Banach space, and let $\{U(t, \tau)\}$ be a multivalued process on $X$. Also let $U(t, \tau) x$ be norm-to-weak upper semicontinuous in $x$ for fixed $t \geqslant \tau, \tau \in \mathbb{R}$; that is, if $x_{n} \rightarrow x$, then for any $y_{n} \in U(t, \tau) x_{n}$, there exist a subsequence $y_{n_{k}} \in U(t, \tau) x_{n_{k}}$ and a $y \in U(t, \tau) x$ such that $y_{n_{k}} \rightarrow y$ (weak convergence). Then the multivalued process $\{U(t, \tau)\}$ possesses a pullback $\mathscr{D}$-attractor $\mathscr{A}=\{A(t)\}_{t \in \mathbb{R}}$ in $X$ given by

$$
\begin{aligned}
A(t) & =\omega_{t}(Q) \\
& =\bigcap_{T \in \mathbb{R}^{+}} \overline{\bigcup_{s \geqslant T} U(t, t-s) Q(t-s)} \subset Q(t)
\end{aligned}
$$

if and only if $\{U(t, \tau)\}$ is pullback $\mathscr{D}$-dissipative and pullback D-limit-set compact with respect to each $t \in \mathbb{R}$, where $\mathbb{Q}=$ $\{Q(t)\}_{t \in \mathbb{R}} \in \mathscr{D}$ is pullback $\mathscr{D}$-absorbing for the multivalued process $\{U(t, \tau)\}$.

A multivalued process $\{U(t, \tau)\}$ is said to be pullback $\mathscr{D}$ asymptotically upper-semicompact in $X$ if for each fixed $t \in$ $\mathbb{R}$, any $\mathscr{B}=\{B(t)\}_{t \in \mathbb{R}} \in \mathscr{D}$, any sequence $\left\{T_{n}\right\}$ with $T_{n} \rightarrow$ $+\infty,\left\{x_{n}\right\}$ with $x_{n} \in B\left(t-T_{n}\right)$, and any $\left\{y_{n}\right\}$ with $y_{n} \in U(t, t-$ $\left.T_{n}\right) x_{n}$; this last sequence $\left\{y_{n}\right\}$ is relatively compact in $X$.

Remark 6. Let $\{U(t, \tau)\}$ be a multivalued process on $X$. Then $\{U(t, \tau)\}$ is pullback $\mathscr{D}$-asymptotically upper-semicompact if and only if $\{U(t, \tau)\}$ is pullback $\mathscr{D}$-limit-set compact; see [21].

Let $X$ be a Banach space, and let $h>0$ be a given positive number (the delay time). Denote by $C_{X}$ the Banach space $C([-h, 0] ; X)$ endowed with the norm

$$
\|\phi\|_{C_{X}}=\sup _{\theta \in[-h, 0]}\|\phi(\theta)\|_{X} .
$$

Let us consider $\mathscr{D}_{C_{X}}$ a class of sets parameterized in time, $\mathscr{D}=\{D(t)\}_{t \in \mathbb{R}} \subset \mathscr{P}\left(C_{X}\right)$. To study the pullback $\mathscr{D}$-limit-set compactness of the multivalued process on $C_{X}$, we need the following result from [20].

Theorem 7. Let $\{U(t, \tau)\}$ be a multivalued process on $C_{X}$. Suppose that for each $t \in \mathbb{R}$, any $\mathscr{B} \in \mathscr{D}_{C_{X}}$ and $\varepsilon>0$, there exist $\tau_{0}=\tau_{0}(t, \mathscr{B}, \varepsilon)>0$, a finite dimensional subspace $X_{1}$ of $X$, and $a \delta>0$ such that

(1) for each fixed $\theta \in[-h, 0]$,

$$
\left\|\bigcup_{s \geqslant \tau_{0}} \bigcup_{u_{t}(\cdot) \in U(t, t-s) B(t-s)} P u(t+\theta)\right\|_{X} \text { is bounded; }
$$

(2) for all $s \geqslant \tau_{0}, u_{t}(\cdot) \in U(t, t-s) B(t-s), \theta_{1}, \theta_{2} \in[-h, 0]$ with $\left|\theta_{2}-\theta_{1}\right|<\delta$,

$$
\left\|P\left(u\left(t+\theta_{1}\right)-u\left(t+\theta_{2}\right)\right)\right\|_{X}<\varepsilon ;
$$

(3) for all $s \geqslant \tau_{0}, u_{t}(\cdot) \in U(t, t-s) B(t-s)$,

$$
\sup _{\theta \in[-h, 0]}\|(I-P) u(t+\theta)\|_{X}<\varepsilon,
$$

where $P: X \rightarrow X_{1}$ is the canonical projector. Then $\{U(t, \tau)\}$ is pullback $\mathscr{D}$-limit-set compact in $C_{X}$ with respect to each $t \in \mathbb{R}$.

\section{Existence of an Absorbing Family of Sets in $C_{V}$}

By the classical Faedo-Galerkin scheme and compactness method, analogous to the arguments in [5], we have the following.

Theorem 8. Let one consider $\phi \in C_{H}, g \in L_{\text {loc }}^{2}(\mathbb{R} ; H)$, and assume that $f: \mathbb{R} \times H \rightarrow H$ satisfies the hypotheses (H1)-(H3). Then, for each $\tau \in \mathbb{R}$,

(a) there exists a weak solution $u$ to problem (9) satisfying $u \in C([\tau-h, T] ; H) \cap L^{\infty}(\tau, T ; H) \cap L^{2}(\tau, T ; V) \quad \forall T \geqslant \tau ;$

(b) if $\phi \in C_{V}$, then there exists a strong solution $u$ to problem (9); that is,

$$
\begin{array}{r}
u \in C([\tau-h, T] ; V) \cap L^{\infty}(\tau, T ; V) \cap L^{2}(\tau, T ; D(A)), \\
\forall T \geqslant \tau .
\end{array}
$$

Given $T>\tau$ and $u:[\tau-h, T) \rightarrow H$, for each $t \in[\tau, T)$, we denote by $u_{t}$ the function defined on $[-h, 0]$ by the relation $u_{t}(s)=u(t+s), s \in[-h, 0]$. We also denote $C_{H}=C([-h, 0]$; $H)$ and $C_{V}=C([-h, 0] ; V)$. Let $C$ be the arbitrary positive constants, which may be different from line to line and even in the same line.

Thanks to Theorem 8 , we can define a multivalued process $\left(C_{V},\{U(\cdot, \cdot)\}\right)$ as

$$
U(t, \tau)(\phi)=\left\{u_{t}(\cdot ; \tau, \phi) \mid u(\cdot)\right. \text { is a strong solution of }
$$

$$
\text { (9) with initial datum } \left.\phi \in C_{V}\right\} \text {. }
$$

We first need a priori estimates for the solution $u$ of (9) in the space $C_{H}$ and a necessary bound on the term $\int_{t-1}^{t} e^{\alpha r}\|u(r)\|^{2} d r$, which will be very useful in our analysis; it relates the absorption property for the multivalued process $\{U(t, \tau)\}$ on $C_{V}$.

Lemma 9. In addition to the assumptions (H1)-(H4), assume that

$$
k_{2}^{2}<\left(\frac{\nu \lambda_{1}}{2}\right)^{2}\left(1-\rho_{*}\right)
$$


holds true. Then

$$
\begin{aligned}
&\left\|u_{t}\right\|_{C_{H}}^{2} \leqslant\left(1+\frac{2 k_{2}^{2} e^{\alpha h}}{\nu \lambda_{1}\left(1-\rho_{*}\right) \alpha}\right) e^{\alpha(\tau-t+h)}\|\phi\|_{C_{H}}^{2} \\
&+\frac{2 k_{1}^{2}}{\nu \lambda_{1} \alpha}+\frac{e^{-\alpha(t-h)}}{2 \varepsilon_{2}} \int_{-\infty}^{t} e^{\alpha s}|g(s)|^{2} d s, \\
& \forall t \geqslant \tau+h, \\
& v \int_{t-1}^{t} e^{\alpha r}\|u(r)\|^{2} d r \\
& \leqslant C e^{\alpha \tau}\|\phi\|_{C_{H}}^{2}+C e^{\alpha t} \\
&+C \int_{-\infty}^{t} e^{\alpha r}|g(r)|^{2} d r, \quad \forall t \geqslant \tau+h+1,
\end{aligned}
$$

provided that $\alpha>0$ is small enough.

Proof. By the energy inequality and the Poincaré inequality, we have

$$
\begin{aligned}
& \frac{d}{d t}|u(t)|^{2}+v \lambda_{1}|u(t)|^{2}+v\|u(t)\|^{2} \\
& \quad \leqslant 2(f(t, u(t-\rho(t))), u(t))+2(g(t), u(t)) .
\end{aligned}
$$

We fixed two positive parameters $\varepsilon_{1}$ and $\varepsilon_{2}$ to be chosen later on. Then by (H3) and Young's inequality, we can deduce that

$$
\begin{array}{r}
|(f(t, u(t-\rho(t))), u(t))| \leqslant|f(t, u(t-\rho(t)))||u(t)| \\
\leqslant \varepsilon_{1}|u(t)|^{2} \\
+\frac{k_{1}^{2}+k_{2}^{2}|u(t-\rho(t))|^{2}}{4 \varepsilon_{1}}, \\
|g(t), u(t)| \leqslant \varepsilon_{2}|u(t)|^{2}+\frac{1}{4 \varepsilon_{2}}|g(t)|^{2} .
\end{array}
$$

Therefore,

$$
\begin{aligned}
\frac{d}{d t}|u(t)|^{2}+\nu\|u(t)\|^{2} \leqslant & \left(2 \varepsilon_{1}+2 \varepsilon_{2}-v \lambda_{1}\right)|u(t)|^{2} \\
& +\frac{k_{1}^{2}+k_{2}^{2}|u(t-\rho(t))|^{2}}{2 \varepsilon_{1}} \\
& +\frac{|g(t)|^{2}}{2 \varepsilon_{2}} .
\end{aligned}
$$

Let $\alpha>0$ to be determined later on. Then it follows that

$$
\begin{aligned}
& \frac{d}{d t}\left(e^{\alpha t}|u(t)|^{2}\right) \\
& =\alpha e^{\alpha t}|u(t)|^{2}+e^{\alpha t} \frac{d}{d t}|u(t)|^{2}
\end{aligned}
$$

$$
\begin{gathered}
\leqslant\left(\alpha+2 \varepsilon_{1}+2 \varepsilon_{2}-v \lambda_{1}\right) e^{\alpha t}|u(t)|^{2}+\frac{e^{\alpha t} k_{1}^{2}}{2 \varepsilon_{1}} \\
+\frac{e^{\alpha t} k_{2}^{2}|u(t-\rho(t))|^{2}}{2 \varepsilon_{1}}+\frac{e^{\alpha t}|g(t)|^{2}}{2 \varepsilon_{2}} .
\end{gathered}
$$

Integrating between $\tau$ and $t(\geqslant \tau)$, we have

$$
\begin{aligned}
e^{\alpha t}|u(t)|^{2} \leqslant & e^{\alpha \tau}|u(\tau)|^{2} \\
& +\left(\alpha+2 \varepsilon_{1}+2 \varepsilon_{2}-\nu \lambda_{1}\right) \int_{\tau}^{t} e^{\alpha s}|u(s)|^{2} d s \\
& +\frac{k_{1}^{2}}{2 \varepsilon_{1}} \int_{\tau}^{t} e^{\alpha s} d s \\
& +\frac{k_{2}^{2}}{2 \varepsilon_{1}} \int_{\tau}^{t} e^{\alpha s}|u(s-\rho(s))|^{2} d s \\
& +\frac{1}{2 \varepsilon_{2}} \int_{\tau}^{t} e^{\alpha s}|g(s)|^{2} d s .
\end{aligned}
$$

Let $r=s-\rho(s)$; note that $\rho(s) \in[0, h]$ and $1 /\left(1-\rho^{\prime}(s)\right) \leqslant$ $1 /\left(1-\rho_{*}\right)$ for all $s \in \mathbb{R}$. Hence,

$$
\begin{aligned}
\frac{k_{2}^{2}}{2 \varepsilon_{1}} \int_{\tau}^{t} e^{\alpha s}|u(s-\rho(s))|^{2} d s & \\
\leqslant & \frac{k_{2}^{2}}{2 \varepsilon_{1}} \frac{1}{1-\rho_{*}} \int_{\tau-h}^{t} e^{\alpha(r+h)}|u(r)|^{2} d r \\
\leqslant & \frac{k_{2}^{2} e^{\alpha h}}{2 \varepsilon_{1}\left(1-\rho_{*}\right)} \\
& \times\left(\int_{\tau-h}^{\tau} e^{\alpha r}|u(r)|^{2} d r+\int_{\tau}^{t} e^{\alpha r}|u(r)|^{2} d r\right) \\
\leqslant & \frac{k_{2}^{2} e^{\alpha(h+\tau)}\|\phi\|_{C_{H}}^{2}}{2 \varepsilon_{1}\left(1-\rho_{*}\right) \alpha} \\
& +\frac{k_{2}^{2} e^{\alpha h}}{2 \varepsilon_{1}\left(1-\rho_{*}\right)} \int_{\tau}^{t} e^{\alpha r}|u(r)|^{2} d r .
\end{aligned}
$$

Combining (30) and (31) together, we get

$$
\begin{aligned}
e^{\alpha t}|u(t)|^{2} \leqslant & \left(1+\frac{k_{2}^{2} e^{\alpha h}}{2 \varepsilon_{1}\left(1-\rho_{*}\right) \alpha}\right) e^{\alpha \tau}\|\phi\|_{C_{H}}^{2} \\
& +\frac{k_{1}^{2} e^{\alpha t}}{2 \varepsilon_{1} \alpha}+\frac{1}{2 \varepsilon_{2}} \int_{-\infty}^{t} e^{\alpha s}|g(s)|^{2} d s \\
& +\left(\alpha+2 \varepsilon_{1}+2 \varepsilon_{2}-\nu \lambda_{1}+\frac{k_{2}^{2} e^{\alpha h}}{2 \varepsilon_{1}\left(1-\rho_{*}\right)}\right) \\
& \times \int_{\tau}^{t} e^{\alpha s}|u(s)|^{2} d s .
\end{aligned}
$$


Let $\varepsilon_{1}=v \lambda_{1} / 4$ and using (23), so we can choose positive constants $\alpha$ and $\varepsilon_{2}$ small enough such that $\alpha+2 \varepsilon_{1}+2 \varepsilon_{2}-$ $\nu \lambda_{1}+\left(k_{2}^{2} e^{\alpha h} / 2 \varepsilon_{1}\left(1-\rho_{*}\right)\right)<0$ and $\alpha<\delta_{0}$ (where $\delta_{0}$ is given in the assumption (H4)). Then, it follows that

$$
\begin{aligned}
e^{\alpha t}|u(t)|^{2} \leqslant & \left(1+\frac{2 k_{2}^{2} e^{\alpha h}}{\nu \lambda_{1}\left(1-\rho_{*}\right) \alpha}\right) e^{\alpha \tau}\|\phi\|_{C_{H}}^{2} \\
& +\frac{2 k_{1}^{2} e^{\alpha t}}{\nu \lambda_{1} \alpha}+\frac{1}{2 \varepsilon_{2}} \int_{-\infty}^{t} e^{\alpha s}|g(s)|^{2} d s .
\end{aligned}
$$

Setting now $t+\theta$ instead of $t$ (where $\theta \in[-h, 0]$ ), multiplying by $e^{-\alpha(t+\theta)}$, it holds

$$
\begin{aligned}
|u(t+\theta)|^{2} \leqslant & \left(1+\frac{2 k_{2}^{2} e^{\alpha h}}{v \lambda_{1}\left(1-\rho_{*}\right) \alpha}\right) e^{\alpha(\tau-t-\theta)}\|\phi\|_{C_{H}}^{2} \\
& +\frac{2 k_{1}^{2}}{v \lambda_{1} \alpha}+\frac{e^{-\alpha(t+\theta)}}{2 \varepsilon_{2}} \int_{-\infty}^{t+\theta} e^{\alpha s}|g(s)|^{2} d s \\
\leqslant & \left(1+\frac{2 k_{2}^{2} e^{\alpha h}}{v \lambda_{1}\left(1-\rho_{*}\right) \alpha}\right) e^{\alpha(\tau-t+h)}\|\phi\|_{C_{H}}^{2} \\
& +\frac{2 k_{1}^{2}}{v \lambda_{1} \alpha}+\frac{e^{-\alpha(t-h)}}{2 \varepsilon_{2}} \int_{-\infty}^{t} e^{\alpha s}|g(s)|^{2} d s .
\end{aligned}
$$

Note that $\left\|u_{t}\right\|_{C_{H}}^{2}=\sup _{\theta \in[-h, 0]}|u(t+\theta)|^{2}$, thus the conclusion (24) follows immediately from (34).

Finally, we will obtain the bound on the term $\nu \int_{t-1}^{t} e^{\alpha r}\|u(r)\|^{2} d r$. It follows from (28) that

$$
\begin{aligned}
v e^{\alpha t}\|u(t)\|^{2} \leqslant & \left(2 \varepsilon_{1}+2 \varepsilon_{2}-v \lambda_{1}\right) e^{\alpha t}|u(t)|^{2} \\
& +\frac{k_{1}^{2} e^{\alpha t}}{2 \varepsilon_{1}}+\frac{k_{2}^{2} e^{\alpha t}|u(t-\rho(t))|^{2}}{2 \varepsilon_{1}} \\
& +\frac{e^{\alpha t}|g(t)|^{2}}{2 \varepsilon_{2}}+\alpha e^{\alpha t}|u(t)|^{2} \\
& -\frac{d}{d t}\left(e^{\alpha t}|u(t)|^{2}\right) .
\end{aligned}
$$

Integrating from $t-1$ to $t$, we have

$$
\begin{aligned}
\nu \int_{t-1}^{t} e^{\alpha r} & \|u(r)\|^{2} d r \\
\leqslant & \left(\alpha+2 \varepsilon_{1}+2 \varepsilon_{2}-v \lambda_{1}\right) \int_{t-1}^{t} e^{\alpha r}|u(r)|^{2} d r \\
& +\frac{k_{1}^{2}}{2 \varepsilon_{1}} \int_{t-1}^{t} e^{\alpha r} d r \\
& +\frac{k_{2}^{2}}{2 \varepsilon_{1}} \int_{t-1}^{t} e^{\alpha r}|u(r-\rho(r))|^{2} d r \\
& +\frac{1}{2 \varepsilon_{2}} \int_{t-1}^{t} e^{\alpha r}|g(r)|^{2} d r \\
& +e^{\alpha(t-1)}|u(t-1)|^{2} .
\end{aligned}
$$

Similar to the arguments of (31), we can deduce that

$$
\begin{aligned}
& \frac{k_{2}^{2}}{2 \varepsilon_{1}} \int_{t-1}^{t} e^{\alpha r}|u(r-\rho(r))|^{2} d r \\
& \leqslant \frac{k_{2}^{2}}{2 \varepsilon_{1}} \frac{1}{1-\rho_{*}} \int_{t-1-h}^{t} e^{\alpha(r+h)}|u(r)|^{2} d r \\
& \leqslant \frac{k_{2}^{2} e^{\alpha h}}{2 \varepsilon_{1}\left(1-\rho_{*}\right)} \\
& \quad \times\left(\int_{t-1-h}^{t-1} e^{\alpha r}|u(r)|^{2} d r+\int_{t-1}^{t} e^{\alpha r}|u(r)|^{2} d r\right) \\
& \leqslant \frac{k_{2}^{2} e^{\alpha(h+t-1)}\left\|u_{t-1}\right\|_{C_{H}}^{2}}{2 \varepsilon_{1}\left(1-\rho_{*}\right) \alpha} \\
& \quad+\frac{k_{2}^{2} e^{\alpha h}}{2 \varepsilon_{1}\left(1-\rho_{*}\right)} \int_{t-1}^{t} e^{\alpha r}|u(r)|^{2} d r .
\end{aligned}
$$

Recall that $\varepsilon_{1}=\nu \lambda_{1} / 4$ and $\alpha+2 \varepsilon_{1}+2 \varepsilon_{2}-\nu \lambda_{1}+\left(k_{2}^{2} e^{\alpha h} / 2 \varepsilon_{1}(1-\right.$ $\left.\left.\rho_{*}\right)\right)<0$. By (24) and (36)-(37), we have (25) as desired, and thus the proof of this lemma is completed.

By slightly modifying the proof of Lemma 1.1 in [23], we have the following result.

Lemma 10. Let $t \in \mathbb{R}$ be given arbitrarily. Let $g$, $h$, and $y$ be three positive locally integrable functions on $(-\infty, t]$ such that $y^{\prime}$ is locally integrable on $(-\infty, t]$, which satisfy that

$$
\begin{gathered}
\frac{d y}{d s} \leqslant g y+h \quad \text { for } s \leqslant t, \\
\int_{t-1}^{t} g(s) d s \leqslant a_{1}, \quad \int_{t-1}^{t} h(s) d s \leqslant a_{2}, \\
\int_{t-1}^{t} y(s) d s \leqslant a_{3},
\end{gathered}
$$

where $a_{1}, a_{2}$, and $a_{3}$ are positive constants. Then

$$
y(t) \leqslant \exp \left(a_{1}\right)\left(a_{3}+a_{2}\right) .
$$

Now we state and prove the main result in this section.

Theorem 11. Suppose in addition to the hypotheses in Lemma 9, assume that

$$
\lim _{t \rightarrow-\infty} \int_{-\infty}^{t} e^{-\gamma(t-r)}|g(r)|^{2} d r<\infty \quad \forall \gamma>0
$$

holds true. Then the multivalued process $\{U(t, \tau)\}$ on $C_{V}$ is pullback D-dissipative.

Proof. We take the inner product of (9) with $A u(t)$, we obtain

$$
\begin{aligned}
\frac{1}{2} \frac{d}{d t}\|u(t)\|^{2}+\nu|A u(t)|^{2}+(B(u(t), u(t)), A u(t)) \\
=(f(t, u(t-\rho(t))), A u(t))+(g(t), A u(t)) .
\end{aligned}
$$


Now we evaluate the terms, using (H3) and Young's inequality, and we arrive to

$$
\begin{aligned}
& |(f(t, u(t-\rho(t))), A u(t))|+|(g(t), A u(t))| \\
& \quad \leqslant \frac{v}{2}|A u(t)|^{2}+\frac{|f(t, u(t-\rho(t)))|^{2}}{v}+\frac{|g(t)|^{2}}{v} \\
& \quad \leqslant \frac{v}{2}|A u(t)|^{2}+\frac{k_{1}^{2}+k_{2}^{2}\left\|u_{t}\right\|_{C_{H}}^{2}}{v}+\frac{|g(t)|^{2}}{v} .
\end{aligned}
$$

Next,

$$
\begin{aligned}
& |(B(u(t), u(t)), A u(t))| \\
& \quad \leqslant C_{1}|u(t)|^{1 / 2}\|u(t)\||A u(t)|^{3 / 2} \\
& \quad \leqslant \frac{v}{4}|A u(t)|^{2}+\frac{C_{2}}{v^{3}}|u(t)|^{2}\|u(t)\|^{4} .
\end{aligned}
$$

Thanks to (41)-(43) and the fact that $\|\varphi\|^{2} \leqslant \lambda_{1}^{-1}|A \varphi|^{2}$ for $\varphi \in D(A)$, we can deduce that

$$
\begin{aligned}
\frac{d}{d t}\|u(t)\|^{2}+\frac{v \lambda_{1}}{2}\|u(t)\|^{2} & \frac{2 k_{1}^{2}+2 k_{2}^{2}\left\|u_{t}\right\|_{C_{H}}^{2}+\frac{2|g(t)|^{2}}{v}}{v} \\
& +\frac{2 C_{2}}{v^{3}}|u(t)|^{2}\|u(t)\|^{4},
\end{aligned}
$$

and consequently,

$$
\begin{gathered}
\frac{d}{d t}\left(e^{\alpha t}\|u(t)\|^{2}\right)+\left(\frac{\nu \lambda_{1}}{2}-\alpha\right) e^{\alpha t}\|u(t)\|^{2} \\
\leqslant \frac{2 k_{1}^{2}+2 k_{2}^{2}\left\|u_{t}\right\|_{C_{H}}^{2}}{\nu} e^{\alpha t}+\frac{2|g(t)|^{2}}{\nu} e^{\alpha t} \\
+\frac{2 C_{2} e^{\alpha t}}{v^{3}}|u(t)|^{2}\|u(t)\|^{4} .
\end{gathered}
$$

Since $\varepsilon_{1}=\nu \lambda_{1} / 4$ and $\alpha+2 \varepsilon_{1}+2 \varepsilon_{2}-\nu \lambda_{1}+\left(k_{2}^{2} e^{\alpha h} / 2 \varepsilon_{1}\left(1-\rho_{*}\right)\right)<$ 0 , it is easy to see that $\left(\nu \lambda_{1} / 2\right)-\alpha>0$. Then

$$
\begin{aligned}
\frac{d}{d t}\left(e^{\alpha t}\|u(t)\|^{2}\right) & \\
\leqslant & \frac{2 k_{1}^{2}+2 k_{2}^{2}\left\|u_{t}\right\|_{C_{H}}^{2}}{v} e^{\alpha t}+\frac{2|g(t)|^{2}}{v} e^{\alpha t} \\
& +\frac{2 C_{2}}{v^{3}}|u(t)|^{2}\|u(t)\|^{2}\|u(t)\|^{2} e^{\alpha t}
\end{aligned}
$$

Let $t \in \mathbb{R}$ be given arbitrarily and taking $\tau$ such that $t \geqslant$ $\tau+h+1$. In order to apply Lemma 10, in view of (24), now we firstly obtain

$$
\begin{aligned}
\int_{t-1}^{t}( & \left.\frac{2 k_{1}^{2}+2 k_{2}^{2}\left\|u_{r}\right\|_{C_{H}}^{2}}{v} e^{\alpha r}+\frac{2|g(r)|^{2}}{v} e^{\alpha r}\right) d r \\
\leqslant & \int_{t-1}^{t} \frac{2 k_{1}^{2}+2 k_{2}^{2}\left\|u_{r}\right\|_{C_{H}}^{2}}{v} e^{\alpha r} d r \\
& +\int_{t-1}^{t} \frac{2|g(r)|^{2}}{v} e^{\alpha r} d r \\
\leqslant & C e^{\alpha t}+C e^{\alpha \tau}\|\phi\|_{C_{H}}^{2}+C \int_{-\infty}^{t} e^{\alpha r}|g(r)|^{2} d r .
\end{aligned}
$$

Then, it follows from (24) and (25) that

$$
\begin{aligned}
& \frac{2 C_{2}}{\nu^{3}} \int_{t-1}^{t}|u(r)|^{2}\|u(r)\|^{2} d r \\
& \leqslant \frac{2 C_{2}}{\nu^{3}} \int_{t-1}^{t}\left\|u_{r}\right\|_{C_{H}}^{2}\|u(r)\|^{2} d r \\
& \leqslant \frac{2 C_{2}}{\nu^{3}} \int_{t-1}^{t}\left(1+\frac{2 k_{2}^{2} e^{\alpha h}}{\nu \lambda_{1}\left(1-\rho_{*}\right) \alpha}\right) e^{\alpha(\tau-r+h)} \\
& \times\|\phi\|_{C_{H}}^{2}\|u(r)\|^{2} d r \\
&+\frac{4 C_{2} k_{1}^{2}}{\nu^{4} \lambda_{1} \alpha} \int_{t-1}^{t}\|u(r)\|^{2} d r \\
&+\frac{2 C_{2}}{\nu^{3}} \int_{t-1}^{t}\left(\frac{e^{-\alpha(r-h)}}{2 \varepsilon_{2}}\|u(r)\|^{2} \int_{-\infty}^{r} e^{\alpha s}|g(s)|^{2} d s\right) d r \\
& \leqslant C e^{2 \alpha \tau} e^{-2 \alpha t}\|\phi\|_{C_{H}}^{2}+C \\
&+C e^{-\alpha t} \int_{-\infty}^{t} e^{\alpha r}|g(r)|^{2} d r+C e^{\alpha \tau} e^{-\alpha t}\|\phi\|_{C_{H}}^{2} \\
&+C e^{\alpha \tau} e^{-2 \alpha t}\|\phi\|_{C_{H}}^{2} \int_{-\infty}^{t} e^{\alpha r}|g(r)|^{2} d r \\
&+C e^{-2 \alpha t}\left(\int_{-\infty}^{t} e^{\alpha r}|g(r)|^{2} d r\right)^{2} .
\end{aligned}
$$

Combining (25) and (47)-(48) together, by Lemma 10, we can conclude that

$$
\|u(t)\|^{2} \leqslant\left(a_{3}+a_{2}\right) e^{a_{1}} \quad \forall t \geqslant \tau+h+1,
$$


where

$$
\begin{aligned}
a_{3}= & C e^{\alpha \tau}\|\phi\|_{C_{H}}^{2}+C e^{\alpha t}+C \int_{-\infty}^{t} e^{\alpha r}|g(r)|^{2} d r, \\
a_{2}= & C e^{\alpha t}+C e^{\alpha \tau}\|\phi\|_{C_{H}}^{2}+C \int_{-\infty}^{t} e^{\alpha r}|g(r)|^{2} d r, \\
a_{1}= & C e^{2 \alpha \tau} e^{-2 \alpha t}\|\phi\|_{C_{H}}^{2}+C \\
& +C e^{-\alpha t} \int_{-\infty}^{t} e^{\alpha r}|g(r)|^{2} d r+C e^{\alpha \tau} e^{-\alpha t}\|\phi\|_{C_{H}}^{2} \\
& +C e^{\alpha \tau} e^{-2 \alpha t}\|\phi\|_{C_{H}}^{2} \int_{-\infty}^{t} e^{\alpha r}|g(r)|^{2} d r \\
& +C e^{-2 \alpha t}\left(\int_{-\infty}^{t} e^{\alpha r}|g(r)|^{2} d r\right)^{2} .
\end{aligned}
$$

Therefore, if we take $\tau$ such that $t \geqslant \tau+1+2 h$, then similar to the above mentioned, we get

$$
\left\|u_{t}\right\|_{C_{V}}^{2}=\sup _{\theta \in[-h, 0]}\|u(t+\theta)\|^{2} \leqslant\left(a_{3}+a_{2}\right) e^{a_{1}} .
$$

We denote by $\mathscr{R}$ the set of all functions $r: \mathbb{R} \rightarrow(0,+\infty)$ such that

$$
\lim _{t \rightarrow-\infty} r^{2}(t)=0
$$

and denote by $\mathscr{D}_{C_{V}}$ the class of all families $\mathscr{D}=\{D(t)\}_{t \in \mathbb{R}} \subset$ $\mathscr{P}\left(C_{V}\right)$ such that $D(t) \subset \overline{\mathcal{N}}\left(0, r_{\mathscr{D}}(t)\right)$, for some $r_{\mathscr{D}} \in \mathscr{R}$, where $\mathscr{P}\left(C_{V}\right)$ denotes the family of all nonempty subsets of $C_{V}$ and $\overline{\mathcal{N}}\left(0, r_{\mathscr{D}}(t)\right)$ denotes the closed ball in $C_{V}$ centered at zero with radius $r_{\mathscr{D}}(t)$.

Denote by $R(t)$ the nonnegative number given for each $t \in \mathbb{R}$ by

$$
\begin{aligned}
(R(t))^{2}= & \left(C e^{\alpha t}+C \int_{-\infty}^{t} e^{\alpha r}|g(r)|^{2} d r\right) \\
& \times \exp \left(C e^{-\alpha t} \int_{-\infty}^{t} e^{\alpha r}|g(r)|^{2} d r\right. \\
& \left.+C e^{-2 \alpha t}\left(\int_{-\infty}^{t} e^{\alpha r}|g(r)|^{2} d r\right)^{2}\right),
\end{aligned}
$$

and consider the family of closed balls $\mathbb{Q}=\{Q(t)\}_{t \in \mathbb{R}}$ in $C_{V}$ defined by

$$
Q(t)=\left\{\psi \in C_{V}:\|\psi\|_{C_{V}} \leqslant R(t)\right\} .
$$

It is straightforward to check that $\mathbb{Q} \in \mathscr{D}_{C_{V}}$, and moreover, by (51) and (52), the family of $\mathbb{Q}$ is pullback $\mathscr{D}$-absorbing for the multivalued process $\{U(t, \tau)\}$ on $C_{V}$.

The proof of Theorem 11 is completed.

\section{Existence of the Pullback Attractors in $C_{V}$}

Theorem 12. Suppose in addition to the hypotheses in Theorem 11 that $g \in C(\mathbb{R} ; H)$. Then there exists a unique pullback $D$-attractor $\left\{A_{C_{V}}(t)\right\}_{t \in R}$ for the multivalued process $\{U(t, \tau)\}$ in $C_{V}$.
Proof. Since $A^{-1}$ is a continuous compact operator in $H$, by the classical spectral theory, there exist a sequence $\left\{\lambda_{j}\right\}_{j=1}^{\infty}$,

$$
0<\lambda_{1} \leqslant \lambda_{2} \leqslant \cdots \leqslant \lambda_{j} \leqslant \cdots, \quad \lambda_{j} \longrightarrow+\infty, \text { as } j \longrightarrow+\infty \text {, }
$$

and a family of elements $\left\{w_{j}\right\}_{j=1}^{\infty}$ of $D(A)$ which are orthonormal in $H$ such that

$$
A w_{j}=\lambda_{j} w_{j} \quad \forall j \in \mathbb{N} .
$$

Let $V_{m}=\operatorname{span}\left\{w_{1}, \ldots, w_{m}\right\}$ in $V$ and $P_{m}: V \rightarrow V_{m}$ be an orthogonal projector.

Let $u=u_{1}+u_{2}$, where $u_{1}=P_{m} u$ and $u_{2}=\left(I-P_{m}\right) u$. We decompose (9) as follows:

$$
\begin{gathered}
\frac{\partial u_{2}(t)}{\partial t}+v A u_{2}(t)+B(u(t), u(t))-P_{m} B\left(u_{1}(t), u_{1}(t)\right) \\
=f(t, u(t-\rho(t))) \\
-P_{m} f\left(t, u_{1}(t-\rho(t))\right)+\left(I-P_{m}\right) g(t) \\
u_{2}(\tau+t)=\left(I-P_{m}\right) \phi(t), \quad t \in[-h, 0] \\
\frac{\partial u_{1}(t)}{\partial t}+v A u_{1}(t)+P_{m} B\left(u_{1}(t), u_{1}(t)\right) \\
\quad=P_{m} f\left(t, u_{1}(t-\rho(t))\right)+P_{m} g(t) \\
u_{1}(\tau+t)=P_{m} \phi(t), \quad t \in[-h, 0] .
\end{gathered}
$$

We divide the proof into three steps.

(1) For every fixed $t \in \mathbb{R}$, any $\mathscr{B}=\{B(t)\}_{t \in \mathbb{R}} \in \mathscr{D}_{C_{V}}$ and $\varepsilon>0$, we observe that for any $T \geqslant t-s$ with $s \geqslant 0$,

$$
\begin{aligned}
& U(T, t-s)(\phi) \\
& =\left\{u_{T}(\cdot ; t-s, \phi) \mid u(\cdot)\right. \text { is a strong solution } \\
& \quad \text { of (9) with } \phi \in B(t-s)\} .
\end{aligned}
$$

Taking the inner product in $H$ of (57) with $A u_{2}=A\left(I-P_{m}\right) u$, we get

$$
\begin{aligned}
\frac{1}{2} \frac{d}{d T} \| & u_{2}(T) \|^{2}+v\left|A u_{2}(T)\right|^{2} \\
\leqslant & \left|\left(f(T, u(T-\rho(T))), A u_{2}(T)\right)\right| \\
& +\left|\left(P_{m} f\left(T, u_{1}(T-\rho(T))\right), A u_{2}(T)\right)\right| \\
& +\left|\left(B(u(T), u(T)), A u_{2}(T)\right)\right| \\
& +\left|\left(P_{m} B\left(u_{1}(T), u_{1}(T)\right), A u_{2}(T)\right)\right| \\
& +\left|\left(\left(I-P_{m}\right) g(T), A u_{2}(T)\right)\right| .
\end{aligned}
$$

By (H3) and Young's inequality, we have

$$
\begin{gathered}
\left|\left(f(T, u(T-\rho(T))), A u_{2}(T)\right)\right|+\left|\left(g(T), A u_{2}(T)\right)\right| \\
\quad \leqslant \frac{v}{8}\left|A u_{2}(T)\right|^{2}+C+C\left\|u_{T}\right\|_{C_{H}}^{2}+C|g(T)|^{2} .
\end{gathered}
$$


To estimate $\left(B(u(T), u(T)), A u_{2}(T)\right)$, we recall some inequalities [19]:

$$
|\varphi|_{\left(L^{\infty}(\Omega)\right)^{2}} \leqslant C_{3}\|\varphi\|\left(1+\log \frac{|A \varphi|^{2}}{\lambda_{1}\|\varphi\|^{2}}\right)^{1 / 2} \quad \forall \varphi \in D(A),
$$

and thus

$$
\begin{aligned}
|B(u, v)| & \leqslant C_{4}|(u \cdot \nabla) v| \leqslant C_{4}|u|_{L^{\infty}(\Omega)}\|v\| \\
& \leqslant C_{4} C_{3}\|u\|\|v\|\left(1+\log \frac{|A u|^{2}}{\lambda_{1}\|u\|^{2}}\right)^{1 / 2} .
\end{aligned}
$$

Note that $\left|A u_{1}\right|^{2} \leqslant \lambda_{m}\left\|u_{1}\right\|^{2}$, and set $L=1+\log \left(\lambda_{m+1} / \lambda_{1}\right)$. Then by Young's inequality, we can deduce that

$$
\begin{aligned}
&\left|\left(B(u(T), u(T)), A u_{2}(T)\right)\right| \\
& \leqslant\left|\left(B\left(u_{2}(T), u_{1}(T)+u_{2}(T)\right), A u_{2}(T)\right)\right| \\
&+\left|\left(B\left(u_{1}(T), u_{1}(T)+u_{2}(T)\right), A u_{2}(T)\right)\right| \\
& \leqslant C_{1}\left|u_{2}(T)\right|^{1 / 2}\left|A u_{2}(T)\right|^{3 / 2} \\
& \times\left(\left\|u_{1}(T)\right\|+\left\|u_{2}(T)\right\|\right) \\
&+C_{3} C_{4} L^{1 / 2}\left\|u_{1}(T)\right\|\left|A u_{2}(T)\right| \\
& \times\left(\left\|u_{1}(T)\right\|+\left\|u_{2}(T)\right\|\right) \\
& \leqslant \frac{v}{8}\left|A u_{2}(T)\right|^{2}+C|u(T)|^{2}\|u(T)\|^{4}+C\|u(T)\|^{4} .
\end{aligned}
$$

By (60)-(64) and Poincaré inequality, we obtain

$$
\begin{aligned}
\frac{d}{d T}\left\|u_{2}(T)\right\|^{2}+v \lambda_{m+1}\left\|u_{2}(T)\right\|^{2} \\
\leqslant C+C\left\|u_{T}\right\|_{C_{H}}^{2}+C|g(T)|^{2} \\
\quad+C|u(T)|^{2}\|u(T)\|^{4}+C\|u(T)\|^{4} .
\end{aligned}
$$

Applying the Gronwall's lemma in the interval $[t-s, t+\theta]$, it yields

$$
\begin{aligned}
& \left\|u_{2}(t+\theta)\right\|^{2} \\
& \leqslant\left\|u_{2}(t-s)\right\|^{2} e^{-v \lambda_{m+1}(\theta+s)} \\
& \quad+C \int_{t-s}^{t+\theta} e^{-\nu \lambda_{m+1}(t+\theta-r)} \\
& \quad \times\left(1+\left\|u_{r}\right\|_{C_{H}}^{2}+|g(r)|^{2}+|u(r)|^{2}\|u(r)\|^{4}+\|u(r)\|^{4}\right) d r .
\end{aligned}
$$

Let $\varepsilon>0$ be given arbitrarily. Note that $g \in C(\mathbb{R} ; H)$, then we can take $m+1$ large enough such that for any fixed $\eta>0$,

$$
\begin{aligned}
& C \int_{t-h-\eta}^{t+\theta} e^{-v \lambda_{m+1}(t+\theta-r)}|g(r)|^{2} d r \leqslant \frac{C}{\nu \lambda_{m+1}}<\frac{\varepsilon}{4} \\
& \sup _{\theta \in[-h, 0]} C \int_{-\infty}^{t-h-\eta} e^{-\nu \lambda_{m+1}(t+\theta-r)}|g(r)|^{2} d r \\
& \leqslant C \int_{-\infty}^{t-h-\eta} e^{-\nu \lambda_{m+1}(t-h-r)}|g(r)|^{2} d r \\
& \leqslant C e^{-v \lambda_{m+1}(t-h)} \\
& \times\left(\int_{t-h-\eta-1}^{t-h-\eta} e^{\nu \lambda_{m+1} r}|g(r)|^{2} d r\right. \\
& \left.+\int_{t-h-\eta-2}^{t-h-\eta-1} e^{\nu \lambda_{m+1} r}|g(r)|^{2} d r+\cdots\right) \\
& \leqslant C e^{-\nu \lambda_{m+1}(t-h)} \\
& \times\left(e^{\left(\nu \lambda_{m+1}-\alpha\right)(t-h-\eta)}+e^{\left(\nu \lambda_{m+1}-\alpha\right)(t-h-\eta-1)}+\cdots\right) \\
& \times \int_{-\infty}^{t} e^{\alpha r}|g(r)|^{2} d r \\
& \leqslant \frac{C e^{-\nu \lambda_{m+1} \eta} e^{-\alpha(t-h-\eta)}}{1-e^{-\left(\nu \lambda_{m+1}-\alpha\right)}} \int_{-\infty}^{t} e^{\alpha r}|g(r)|^{2} d r \\
& <\frac{\varepsilon}{4} \text {. }
\end{aligned}
$$

Combining (67) and (68) together, we can get for $m+1$ large enough,

$$
\sup _{\theta \in[-h, 0]} C \int_{-\infty}^{t+\theta} e^{-v \lambda_{m+1}(t+\theta-r)}|g(r)|^{2} d r<\frac{\varepsilon}{2}
$$

On the other hand, thanks to Lemma 9 and Theorem 11, we can deduce that when $m+1$ and $s$ are large enough,

$$
\begin{aligned}
& \sup _{\theta \in[-h, 0]}\left\|u_{2}(t-s)\right\|^{2} e^{-\nu \lambda_{m+1}(\theta+s)} \\
& \leqslant\left\|u_{2}(t-s)\right\|^{2} e^{-v \lambda_{m+1}(s-h)}<\frac{\varepsilon}{4}, \\
& \sup _{\theta \in[-h, 0]} C \int_{t-s}^{t+\theta} e^{-\nu \lambda_{m+1}(t+\theta-r)} \\
& \quad \times\left(C+\left\|u_{r}\right\|_{C_{H}}^{2}+|u(r)|^{2}\|u(r)\|^{4}+\|u(r)\|^{4}\right) d r \\
& <\frac{\varepsilon}{4} .
\end{aligned}
$$


Thanks to (69) and (70), it follows from (66) that when $m+1$ and $s$ are large enough,

$$
\begin{aligned}
& \left\|u_{2 t}\right\|_{C_{V}}^{2} \\
& =\sup _{\theta \in[-h, 0]}\left\|u_{2}(t+\theta)\right\|^{2} \\
& \leqslant \sup _{\theta \in[-h, 0]}\left\|u_{2}(t-s)\right\|^{2} e^{-\nu \lambda_{m+1}(\theta+s)} \\
& +\sup _{\theta \in[-h, 0]} C \int_{t-s}^{t+\theta} e^{-\nu \lambda_{m+1}(t+\theta-r)} \\
& \quad \times\left(C+\left\|u_{r}\right\|_{C_{H}}^{2}+|g(r)|^{2}+|u(r)|^{2}\|u(r)\|^{4}\right. \\
& \left.+\|u(r)\|^{4}\right) d r
\end{aligned}
$$$$
<\varepsilon \text {. }
$$

(2) Now we consider the ordinary functional differential system (58) and check the condition (2) in Theorem 7. Note that $\left|A u_{1}\right|^{2} \leqslant \lambda_{m}\left\|u_{1}\right\|^{2} \leqslant \lambda_{m}^{2}\left|u_{1}\right|^{2}$. Without generality, we assume that $\theta_{1}, \theta_{2} \in[-h, 0]$ with $0<\theta_{1}-\theta_{2}<1$. Hence

$$
\begin{aligned}
& \left\|u_{1}\left(t+\theta_{1}\right)-u_{1}\left(t+\theta_{2}\right)\right\| \\
& \leqslant \sqrt{\lambda_{m}}\left|u_{1}\left(t+\theta_{1}\right)-u_{1}\left(t+\theta_{2}\right)\right| \\
& \leqslant \sqrt{\lambda_{m}} \int_{t+\theta_{2}}^{t+\theta_{1}}\left|\frac{d u_{1}(T)}{d T}\right| d T \\
& \leqslant \sqrt{\lambda_{m}} \int_{t+\theta_{2}}^{t+\theta_{1}}\left(\nu\left|A u_{1}(T)\right|+\left|B\left(u_{1}(T), u_{1}(T)\right)\right|\right. \\
& \left.\quad+\left|f\left(T, u_{1}(T-\rho(T))\right)\right|+\left|P_{m} g(T)\right|\right) d T .
\end{aligned}
$$

Notice that

$$
\left|B\left(u_{1}, u_{1}\right)\right| \leqslant C\left|A u_{1}\right|\left\|u_{1}\right\| \leqslant C \sqrt{\lambda_{m}}\left\|u_{1}\right\|^{2} \leqslant C \lambda_{m}^{3 / 2}\left|u_{1}\right|^{2} .
$$

Then, it follows from (H3), (H4), and (24) that

$$
\begin{aligned}
& \int_{t+\theta_{2}}^{t+\theta_{1}}\left(\left|A u_{1}(T)\right|+\left|B\left(u_{1}(T), u_{1}(T)\right)\right|\right) d T \\
& \leqslant C \int_{t+\theta_{2}}^{t+\theta_{1}}\left|u_{1}(T)\right| d T+C \int_{t+\theta_{2}}^{t+\theta_{1}}\left|u_{1}(T)\right|^{2} d T \\
& \leqslant C \int_{t+\theta_{2}}^{t+\theta_{1}}\left|u_{1}(T)\right|^{2} d T+C\left(\theta_{1}-\theta_{2}\right) \\
& \leqslant C\left(e^{-\alpha \theta_{2}}-e^{-\alpha \theta_{1}}\right)+C\left(\theta_{1}-\theta_{2}\right) \\
& \quad+C\left(e^{-\alpha \theta_{2}}-e^{-\alpha \theta_{1}}\right) e^{-\alpha t} \int_{-\infty}^{t} e^{\alpha r}|g(r)|^{2} d r,
\end{aligned}
$$

$$
\begin{aligned}
& \int_{t+\theta_{2}}^{t+\theta_{1}}\left|f\left(T, u_{1}(T-\rho(T))\right)\right| d T \\
& \leqslant \int_{t+\theta_{2}}^{t+\theta_{1}}\left(\left|f\left(T, u_{1}(T-\rho(T))\right)\right|^{2}+C\right) d T \\
& \leqslant \int_{t+\theta_{2}}^{t+\theta_{1}}\left(k_{2}^{2}\left\|u_{1 T}\right\|_{C_{H}}^{2}+C\right) d T \\
& \leqslant C\left(e^{-\alpha \theta_{2}}-e^{-\alpha \theta_{1}}\right)+C\left(\theta_{1}-\theta_{2}\right) \\
& \quad+C\left(e^{-\alpha \theta_{2}}-e^{-\alpha \theta_{1}}\right) e^{-\alpha t} \int_{-\infty}^{t} e^{\alpha r}|g(r)|^{2} d r .
\end{aligned}
$$

Since $g \in C(\mathbb{R} ; H)$ and $t$ is fixed,

$$
\int_{t+\theta_{2}}^{t+\theta_{1}}\left|P_{m} g(T)\right| d T \leqslant C\left(\theta_{1}-\theta_{2}\right) .
$$

Equations (74)-(75) imply that the condition (2) in Theorem 7 is proved.

(3) Invoking Theorem 7, in view of the previous arguments and Theorem 11, we can see that the multivalued process $\{U(t, \tau)\}$ is pullback $\mathscr{D}$-limit-set compact and pullback $\mathscr{D}$-dissipative in $C_{V}$.

In order to get the existence of pullback $\mathscr{D}$-attractors, by the proof of Theorem 3.2 in [21], now we only need to show the negative invariance of $\left\{A_{C_{V}}(t)\right\}_{t \in \mathbb{R}}$, where

$$
\begin{aligned}
A_{C_{V}}(t) & =\omega_{t}(\mathbb{Q}) \\
& =\bigcap_{T \in \mathbb{R}^{+}} \overline{\bigcup_{s \geqslant T} U(t, t-s) Q(t-s)}, \quad \forall t \in \mathbb{R},
\end{aligned}
$$

and $\mathbb{Q}=\{Q(t)\}_{t \in \mathbb{R}} \in \mathscr{D}_{C_{V}}$ is a pullback $\mathscr{D}$-absorbing set of $\{U(t, \tau)\}$ in $C_{V}$.

Let $y \in A_{C_{Y}}(t)$. Then there exist sequences $s_{n} \in \mathbb{R}^{+}, s_{n} \rightarrow$ $+\infty(n \rightarrow \infty), x_{n} \in Q\left(t-s_{n}\right)$, and $y_{n} \in U\left(t, t-s_{n}\right) x_{n}$ such that

$$
y_{n} \longrightarrow y \text { in } C_{V} \text { as } n \longrightarrow \infty .
$$

On the other hand, for $n$ sufficiently large,

$$
y_{n} \in U\left(t, t-s_{n}\right) x_{n}=U(t, \tau) U\left(\tau, t-s_{n}\right) x_{n} .
$$

Then by the pullback $\mathscr{D}$-limit-set compactness of the multivalued process $\{U(t, \tau)\}$, there is a subsequence of $\tilde{x}_{n} \in$ $U\left(\tau, t-s_{n}\right) x_{n}=U\left(\tau, \tau-\left(\tau+s_{n}-t\right)\right) x_{n}$, which we still relabel as $\widetilde{x}_{n}$ such that $y_{n} \in U(t, \tau) \widetilde{x}_{n}$ and

$$
\tilde{x}_{n} \longrightarrow x \quad \text { in } C_{V} \text { as } n \longrightarrow \infty \text {. }
$$

Clearly, $x \in A_{C_{V}}(\tau)$.

We observe that $y_{n}$ is bounded in $C_{V}$ for $n$ sufficiently large. Then by slightly modifying the proof of the existence of solutions (see [16] for details), in view of Theorem 2.11 in [21], we can see that

$$
y_{n}(\cdot)-u(\cdot+t, \tau, x) \quad \text { in } L^{2}([-h, 0] ; V) .
$$

This together with (77)-(79), we can deduce that $y \in$ $U(t, \tau) x \subset U(t, \tau) A_{C_{V}}(\tau)$, and thus the proof of Theorem 12 is finished. 


\section{Acknowledgments}

This research was supported by the National Natural Science Foundation of China under Grant no. 10801066 and the Fundamental Research Funds for the Central Universities under Grant no. lzujbky-2011-47 and no. lzujbky-2012-k26. The Project was sponsored by the Scientific Research Foundation for the Returned Overseas Chinese Scholars, State Education Ministry.

\section{References}

[1] P. Constantin and C. Foias, Navier-Stokes Equations, The University of Chicago Press, Chicago, Ill, USA, 1988.

[2] P. Marín-Rubio and J. C. Robinson, "Attractors for the stochastic 3D Navier-Stokes equations," Stochastics and Dynamics, vol. 3, no. 3, pp. 279-297, 2003.

[3] R. Temam, Navier-Stokes Equations: Theory and Numerical Analysis, vol. 2, North-Holland, Amsterdam, The Netherlands, 2nd edition, 1979.

[4] T. Caraballo and J. Real, "Attractors for 2D-Navier-Stokes models with delays," Journal of Differential Equations, vol. 205, no. 2, pp. 271-297, 2004.

[5] P. Marín-Rubio and J. Real, "Pullback attractors for 2D-NavierStokes equations with delays in continuous and sub-linear operators," Discrete and Continuous Dynamical Systems A, vol. 26, no. 3, pp. 989-1006, 2010.

[6] T. Caraballo, P. Marín-Rubio, and J. Valero, "Autonomous and non-autonomous attractors for differential equations with delays," Journal of Differential Equations, vol. 208, no. 1, pp. 9-41, 2005.

[7] T. Caraballo, P. Marín-Rubio, and J. Valero, "Attractors for differential equations with unbounded delays," Journal of Differential Equations, vol. 239, no. 2, pp. 311-342, 2007.

[8] J. K. Hale and S. M. Verduyn-Lunel, Introduction to Functional Differential Equations, Springer, Berlin, Germany, 1993.

[9] J. Mallet-Paret and G. R. Sell, "Systems of differential delay equations: floquet multipliers and discrete Lyapunov functions," Journal of Differential Equations, vol. 125, no. 2, pp. 385-440, 1996.

[10] J. Mallet-Paret and G. R. Sell, "The Poincaré-Bendixson theorem for monotone cyclic feedback systems with delay," Journal of Differential Equations, vol. 125, no. 2, pp. 441-489, 1996.

[11] T. Caraballo, M. J. Garrido-Atienza, B. Schmalfuß, and J. Valero, "Non-autonomous and random attractors for delay random semilinear equations without uniqueness," Discrete and Continuous Dynamical Systems A, vol. 21, no. 2, pp. 415-443, 2008.

[12] T. Caraballo and P. E. Kloeden, "Non-autonomous attractor for integro-differential evolution equations," Discrete and Continuous Dynamical Systems S, vol. 2, no. 1, pp. 17-36, 2009.

[13] T. Caraballo, P. E. Kloeden, and J. Real, "Pullback and forward attractors for a damped wave equation with delays," Stochastics and Dynamics, vol. 4, no. 3, pp. 405-423, 2004.

[14] T. Caraballo, G. Łukaszewicz, and J. Real, "Pullback attractors for asymptotically compact non-autonomous dynamical systems," Nonlinear Analysis: Theory, Methods and Applications A, vol. 64, no. 3, pp. 484-498, 2006.

[15] D. N. Cheban, P. E. Kloeden, and B. Schmalfuß, "The relationship between pullback, forward and global attractors of nonautonomous dynamical systems," Nonlinear Dynamics and Systems Theory, vol. 2, no. 2, pp. 9-28, 2002.

[16] V. V. Chepyzhov and M. I. Vishik, Attractors for Equations of Mathematical Physics, vol. 49, American Mathematical Society, Providence, RI, USA, 2002.

[17] P. E. Kloeden and B. Schmalfuß, "Asymptotic behaviour of nonautonomous difference inclusions," Systems \& Control Letters, vol. 33, no. 4, pp. 275-280, 1998.

[18] P. E. Kloeden and J. A. Langa, "Flattening, squeezing and the existence of random attractors," Proceedings of the Royal Society of London A, vol. 463, no. 2077, pp. 163-181, 2007.

[19] Y. Wang, C. Zhong, and S. Zhou, "Pullback attractors of nonautonomous dynamical systems," Discrete and Continuous Dynamical Systems A, vol. 16, no. 3, pp. 587-614, 2006.

[20] Y. J. Wang and P. E. Kloeden, “The uniform attractor of a multivalued process generated by reaction-diffusion delay equations on an unbounded domain," Discrete and Continuous Dynamical Systems A. In press.

[21] Y. Wang and S. Zhou, "Kernel sections on multi-valued processes with application to the nonlinear reaction-diffusion equations in unbounded domains," Quarterly of Applied Mathematics, vol. 67, no. 2, pp. 343-378, 2009.

[22] C.-K. Zhong, M.-H. Yang, and C.-Y. Sun, "The existence of global attractors for the norm-to-weak continuous semigroup and application to the nonlinear reaction-diffusion equations," Journal of Differential Equations, vol. 223, no. 2, pp. 367-399, 2006.

[23] R. Temam, Infinite Dimensional Dynamical Systems in Mechanics and Physics, Springer, New York, NY, USA, 1988. 


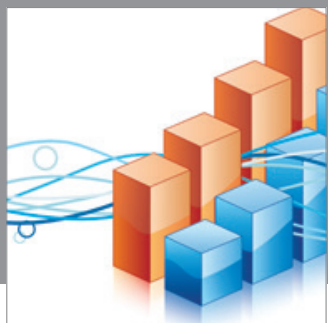

Advances in

Operations Research

mansans

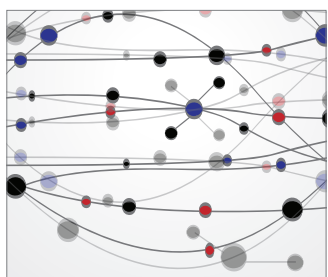

The Scientific World Journal
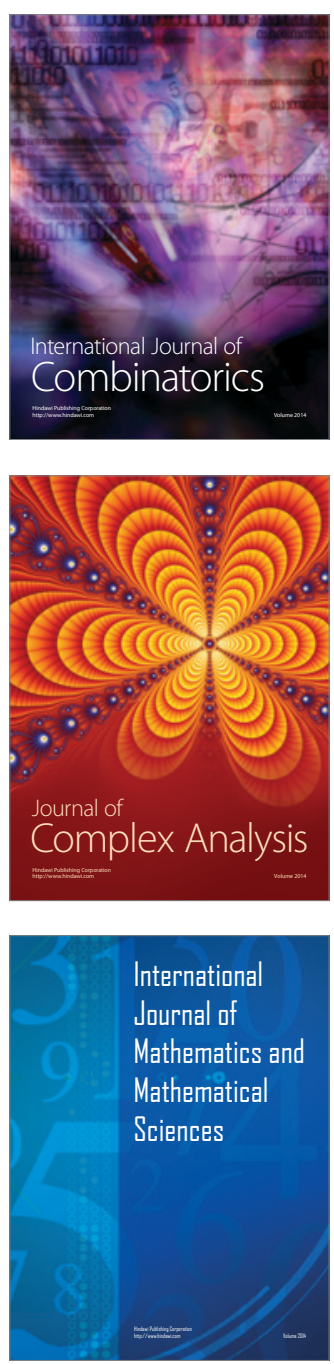
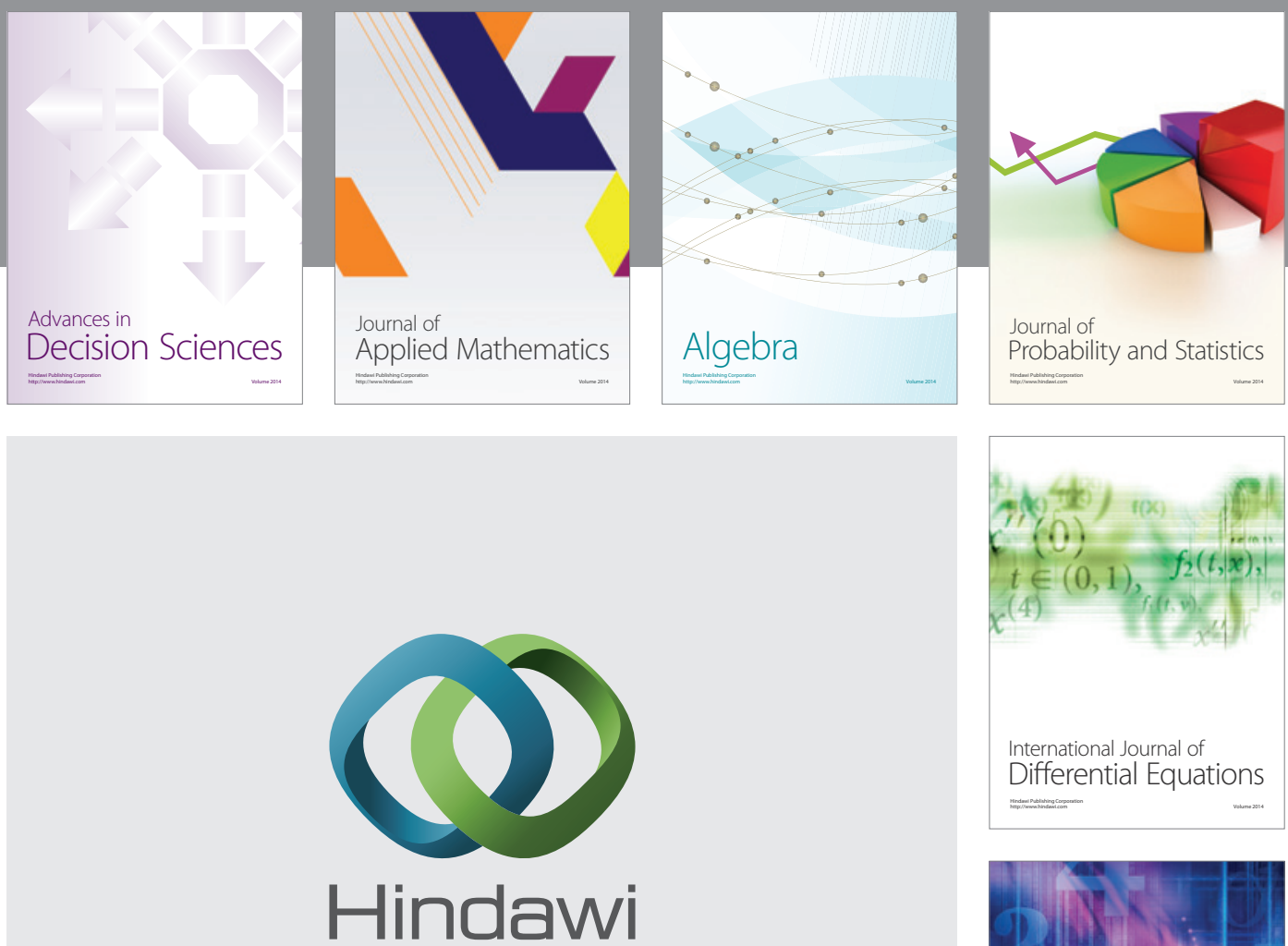

Submit your manuscripts at http://www.hindawi.com
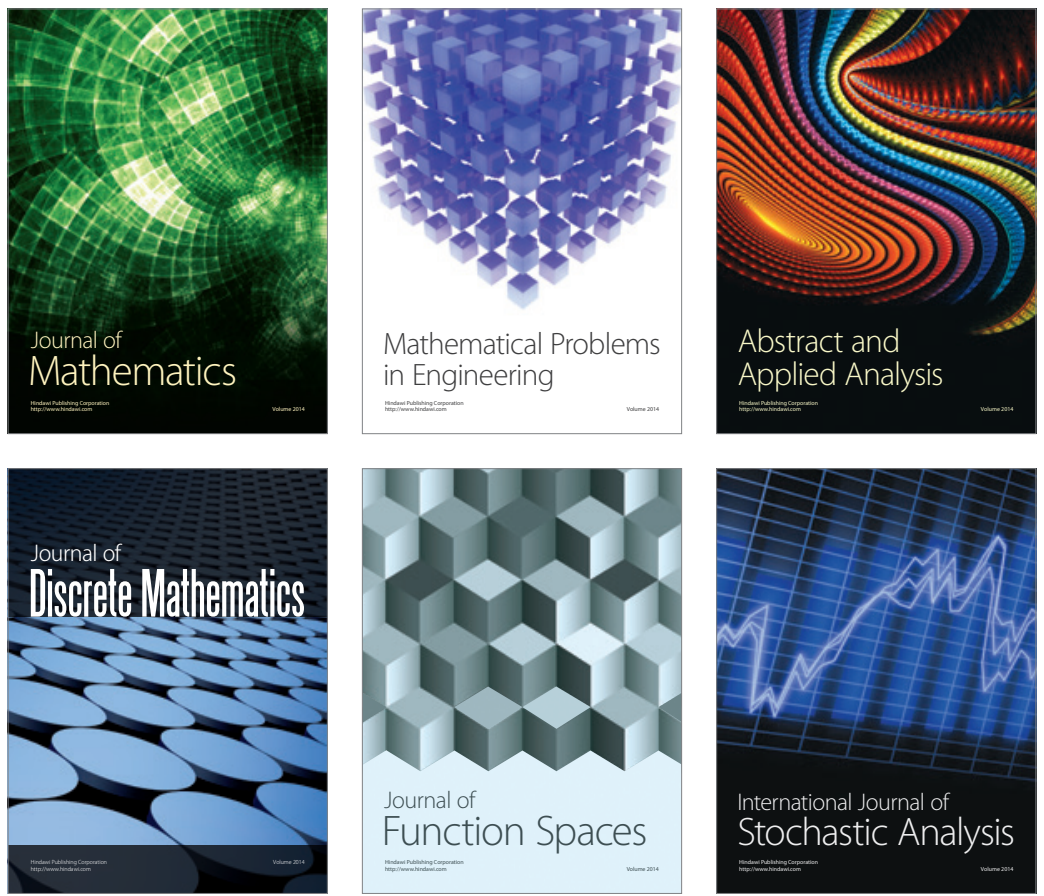

Journal of

Function Spaces

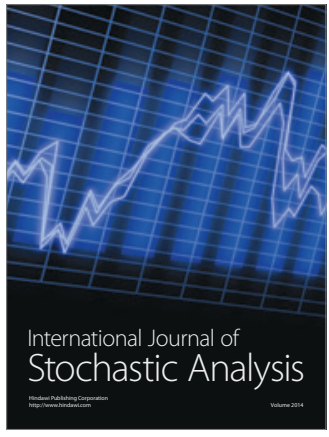

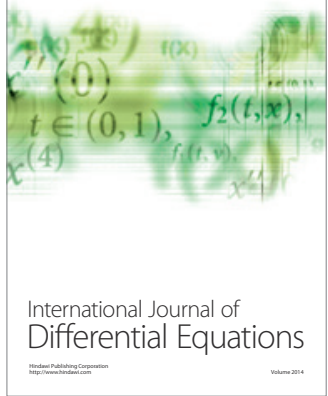
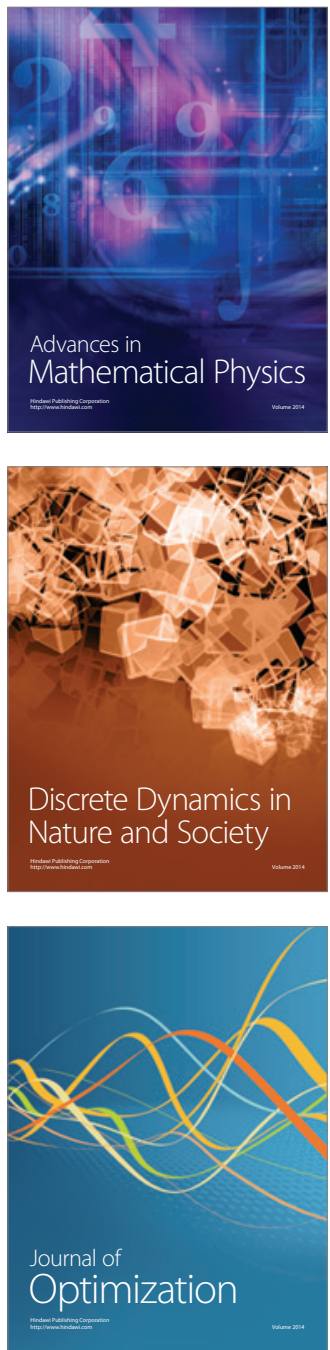